\title{
Recent pulsar results from VERITAS on Geminga and the missing link binary pulsar PSR J1023+0038
}

\author{
G. T. Richards for the VERITAS Collaboration* \\ Georgia Institute of Technology \\ E-mail: gtrichards@gatech.edu
}

\begin{abstract}
In recent years, the Fermi-LAT gamma-ray telescope has detected a population of over 160 gamma-ray pulsars, which has enabled the detailed study of electromagnetic radiation from pulsars at energies above $100 \mathrm{MeV}$. Further, since the surprising detection of the Crab pulsar in very high-energy (VHE; E > $100 \mathrm{GeV}$ ) gamma rays by the MAGIC and VERITAS collaborations, there has been an ongoing effort in the astrophysics community to detect new pulsars in the VHE band. However, the Crab remains the only pulsar so far detected in VHE gamma rays, raising the question of whether or not the Crab is unique and also making it more difficult to constrain model predictions that attempt to explain the emission. Presented here are recent VERITAS results from observational campaigns on the brightest northern-hemisphere high-energy gamma-ray pulsar Geminga and the missing link binary pulsar PSR J1023+0038, which have both resulted in upper limits on a possible VHE flux. These limits are placed into context with the current theoretical framework attempting to explain the origin of the gamma-ray emission from pulsars. Additionally, future plans for pulsar observations with VERITAS will be briefly discussed.
\end{abstract}

The 34th International Cosmic Ray Conference,

30 July- 6 August, 2015

The Hague, The Netherlands

\footnotetext{
*veritas.sao.arizona.edu

${ }^{\dagger}$ Speaker.
} 


\section{Introduction}

The field of pulsar astronomy has entered a new era in the time since the launch of the Fermi Large Area Telescope (LAT), which has now succeeded in detecting over 160 pulsars in the highenergy (HE; E $>100 \mathrm{MeV}$ ) gamma-ray band ${ }^{1}$. These detections have greatly facilitated the detailed study of electromagnetic radiation from pulsars at the highest energies. The spectral energy distributions (SEDs) that have been seen for pulsars in gamma rays are all well-characterized by a broad curvature radiation component that originates due to the electrons and positrons that fill the magnetosphere and follow curved trajectories as they are confined to magnetic field lines. A curvature radiation component has a natural end where the emission becomes radiation-reaction limited [1], resulting in a spectral break at a few $\mathrm{GeV}$ [2]. The spectral "tails" of pulsars in the GeV band are well-described by an exponential cutoff as expected for curvature radiation [2], though statistics are sparse above $\sim 10 \mathrm{GeV}$ due to the decreasing sensitivity of the Fermi-LAT at these high energies.

In recent years, a few pulsars have been detected at energies considerably above the HE spectral break. The Vela pulsar, the brightest steady gamma-ray source seen by the Fermi-LAT [3], has been detected from the ground by H.E.S.S. ${ }^{2}$ above $30 \mathrm{GeV}$ (Gajdus, M., these proceedings) [4] and by the Fermi-LAT above $50 \mathrm{GeV}$ [5]. The H.E.S.S. detection of the Vela pulsar makes it the second pulsar to have been detected in gamma rays from the ground. The first, the Crab pulsar, has been detected in the VHE band above $100 \mathrm{GeV}$ by MAGIC [6] and VERITAS [7] and has posed a challenge in that the curvature radiation scenario is not adequate for a full explanation of the gamma-ray radiation; the combined Fermi-LAT and VERITAS SED favors a power-law fit above $\sim 10 \mathrm{GeV}$ [7]. Current models that have been proposed to explain the VHE emission from the Crab pulsar include inverse-Compton (IC) scattering scenarios in the outer magnetosphere $[8,9,10]$ and beyond the light cylinder [11, 12], and synchrotron radiation from magnetic reconnection events [13]. Whether or not the VHE emission from the Crab pulsar is exceptional with respect to the rest of the gammaray pulsar population remains to be seen.

In this paper we review recent results from the VERITAS collaboration on the Geminga pulsar and PSR J1023+0038. The paper is structured in the following way: in Sections 1.1 and 1.2 we review the Geminga pulsar and PSR J1023+0038, respectively; in Section 2 we discuss the VERITAS observations, analyses, and main results; and in Section 3 we present a brief overview of future pulsar science plans with VERITAS.

\subsection{The Geminga Pulsar}

Formally known as PSR J0633+1746, the Geminga pulsar is located at the relatively close distance of $\sim 200 \mathrm{pc}[14,15]$ and is the second-brightest steady HE gamma-ray source in the sky. The Geminga pulsar has a spin period of $\sim 240 \mathrm{~ms}$ and a spin-down luminosity of $3.2 \times 10^{34} \mathrm{erg}$ $\mathrm{s}^{-1}$ [16]. It is the first GeV-emitting pulsar detected with no known radio counterpart [17] and was, in fact, first discovered in gamma rays by the $S A S-2$ [18] and COS-B [19] satellites. Though radio searches have failed to reveal any hints of emission, the Geminga pulsar is detected in UV [20] and X-rays [21]. Similar to the Crab pulsar in the high-energy band, Geminga shows a doublepeaked phaseogram separated by a "bridge" of emission above the background, with only one of

\footnotetext{
${ }^{1}$ https://confluence.slac.stanford.edu/display/GLAMCOG/Public+List+of+LAT-Detected+Gamma-R ay+Pulsars/

${ }^{2}$ http://www.mpg.de/8287998/velar_pulsar/
} 
the peaks remaining dominant above about $10 \mathrm{GeV}$. As has been seen for other gamma-ray pulsars, the spectrum of Geminga above $100 \mathrm{MeV}$ is can be described by a power law with an exponential cut-off [2], characteristic of the curvature radiation processes thought to dominate in the magnetosphere. However, [10] reported that the spectrum is better described by a simple power-law above the break energy, bringing into question that the spectrum is best described by the exponential cut-off expected in a curvature radiation scenario.

\subsection{PSR J1023+0038}

The system PSR J1023+0038 is an eclipsing binary system located at a distance of $1370 \pm$ $40 \mathrm{pc}$ [22] containing a millisecond pulsar (MSP) with a rapid rotation period of $1.69 \mathrm{~ms}$ orbiting a non-degenerate companion star every $\sim 5 \mathrm{hr}$, found in a survey in 2008 by the Green Bank Telescope [23]. Before the discovery of the MSP, the system was first identified as a low-mass X-ray binary (LMXB) with an accretion disk [24] in 2001. Later, optical and X-ray observations revealed the disappearance of the accretion disk, implying a change to the MSP state [25], though the MSP was not identified until 2008. However, in 2013 June the radio pulsations disappeared [26], and the system was shown to have reformed an accretion disk [27], thus reverting back to the LMXB phase and constituting the first time a system has been seen to oscillate between the two states. Furthermore, it has been thought that MSPs are old pulsars that are spun up to rapid rotation frequencies through the accretion of material from a companion star [28] in a process sometimes referred to as "recycling," and the observed behavior of the so-called "missing-link" PSR J1023+0038 system has helped solidify the recycling scenario as the preferred explanantion for the origin rapid rotation of MSPs. To our knowledge, this work represents the first time that this intriguing system has probed for possible VHE gamma-ray emission.

\section{Observations, Analysis, and Results}

The VERITAS array of four $12 \mathrm{~m}$ diameter imaging atmospheric Cherenkov telescopes is located at the Fred Lawrence Whipple Observatory (FLWO) in southern Arizona ( $31^{\circ} 40^{\prime} \mathrm{N}, 110^{\circ}$ $57^{\prime} \mathrm{W}, 1.3 \mathrm{~km}$ a.s.1.) and started full array operations in 2007 . The telescope reflectors each consist of 345 hexagonal mirror facets, and the cameras comprise 499 photomultiplier tubes giving a total field of view of $\sim 3.5^{\circ}$. VERITAS is sensitive to gamma-ray photons in the energy range 0.85 to $>30 \mathrm{TeV}$ with a sensitivity to detect a $1 \%$ Crab Nebula source in $\sim 25 \mathrm{hr}$. It has an energy resolution of $15-25 \%$, an angular resolution of $0.1^{\circ}$ at $68 \%$ containment, and a pointing accuracy error of less than 50 arcseconds [29]. 


\subsection{Geminga}

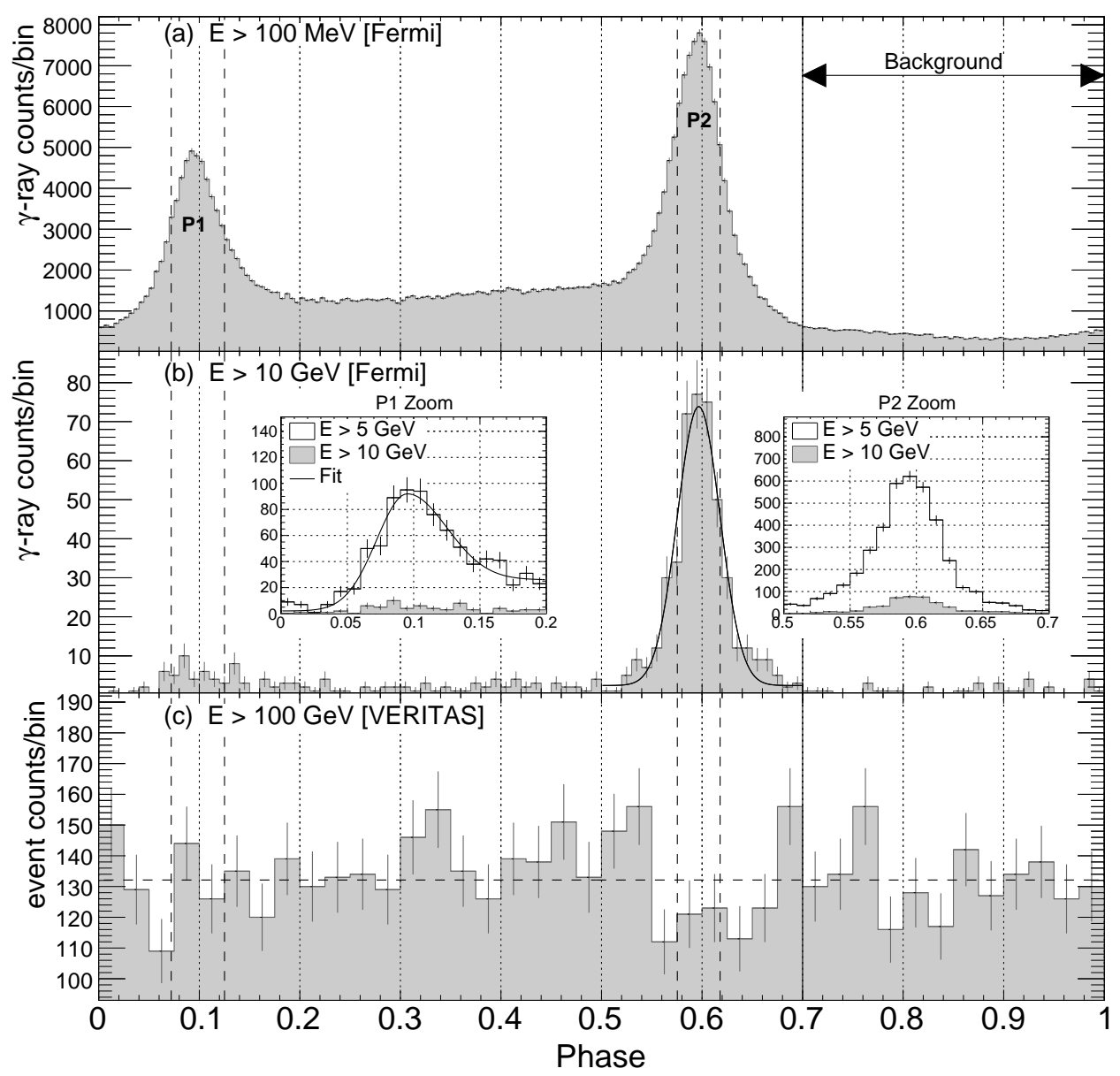

Figure 1: Phase-folded light curves for the Geminga pulsar for both the Fermi-LAT (panels a and b) and VERITAS data (panel c). In panel a, the phase-folded light curve of the Fermi-LAT data with an energy threshold of $\mathrm{E}>100 \mathrm{MeV}$ is shown, and the region defined as background is indicated by the arrow. Panel $\mathrm{b}$ shows the data with a higher energy threshold of $10 \mathrm{GeV}$, and the asymmetric Gaussian fit to P2 is represented by the solid black curve. The inset zoom panels for P1 and P2 show the respective peaks with energy thresholds of both 5 and $10 \mathrm{GeV}$, with the asymmetric Guassian fit to P1 represented by the solid black curve. Panel c shows the phase-folded VERITAS data from the location of the Geminga pulsar in units of counts per bin, and the average number of counts is indicated by the horizontal dashed line. The vertical dashed lines in all three panels represent the phase regions for P1 and P2. Figure from [31].

The VERITAS observational campaign on Geminga has resulted in the accumulation of a total of $71.6 \mathrm{hr}$ of quality-selected data. Event arrival times are barycentered and phase-folded with Tempo2 [30] using an XMM-Newton timing solution (E. Gotthelf 2014, private communication) for data obtained before the launch of the Fermi-LAT. A publically available Fermi-LAT ephemeris from a webpage maintained by M. Kerr ${ }^{3}$ is used for phase-folding all other VERITAS data. The phase-folded Geminga pulsar data recorded by the Fermi-LAT shows two emission peaks (P1 and

\footnotetext{
${ }^{3}$ www.slac.stanford.edu/ kerrm/fermi_pulsar_timing/
} 
P2), which are used to define phase regions of expected signal for the VHE gamma-ray data taken by VERITAS. The peaks P1 and P2 are both fitted with asymmetric Gaussian functions above 5 and $10 \mathrm{GeV}$, respectively, which allows measuring the widths of the pulses at the highest energies possible in the Fermi-LAT band. The two expected VHE signal regions are defined a priori as the $\pm 1 \sigma$ regions for $\mathrm{P} 1$ and $\mathrm{P} 2$ ([0.072-0.125] and [0.575-0.617], respectively), and the background region is defined as [0.7-1.0] [31]. The phase-folded Fermi-LAT and VERITAS light curves are shown in Figure 1.

The test for a pulsed signal using the $\pm 1 \sigma$ regions defined above reveals no evidence pulsed emission from the Geminga pulsar in the VERITAS data. To more generally test for periodicity in the phase-folded VERITAS data, the $H$-Test [32] is employed. The computed $H$ statistic is 1.8 , corresponding to a random chance probability of 0.49 , indicating that phase-folded VERITAS data is consistent with a random distribution. Upper limits (ULs) on excess counts for the P1 and P2 phase regions are computed at the 95\% confidence level (CL) using the method of [33]. These limits are converted to integral flux ULs above $135 \mathrm{GeV}$ of $4.0 \times 10^{-13} \mathrm{~cm}^{-2} \mathrm{~s}^{-1}$ for P1 and $1.7 \times 10^{-13} \mathrm{~cm}^{-2} \mathrm{~s}^{-1}$ for P2. These limits are shown along with a phase-averaged Fermi-LAT SED for the Geminga pulsar in Figure 2. For a more complete explanation of this analysis and the results, see the recent VERITAS publication on the Geminga pulsar [31].

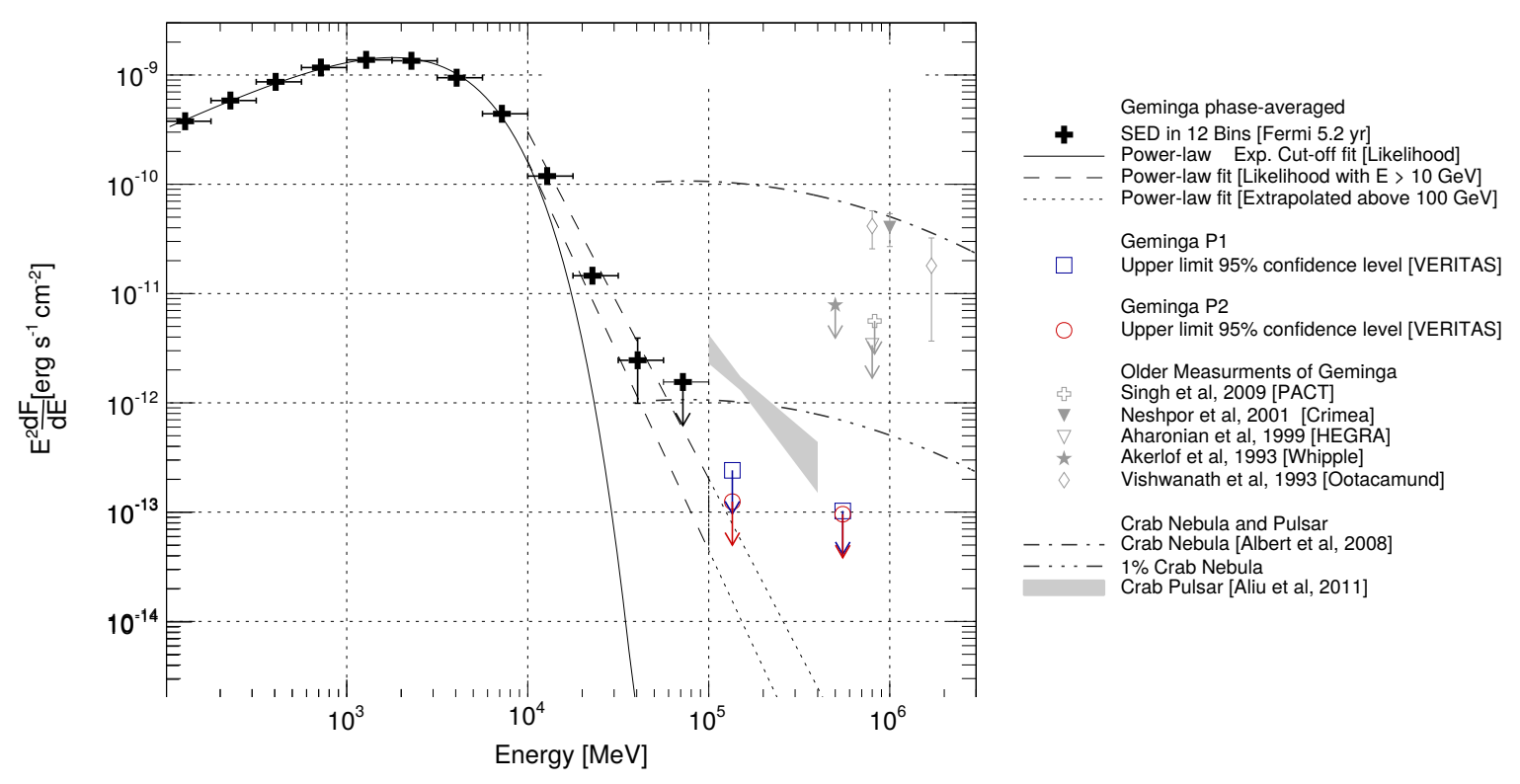

Figure 2: Phase-averaged SED for the Geminga pulsar derived from 5.2 yr of Fermi-LAT data with VHE flux ULs (open squares and circles) for the P1 and P2 phase regions computed from the VERITAS data. The energy thresholds for the two sets of VHE flux ULs are 135 and $550 \mathrm{GeV}$. For a full description of the Fermi-LAT data analysis, see the recent VERITAS collaboration publication on the Geminga pulsar [31]. 


\subsection{PSR J1023+0038}

\begin{tabular}{cccc}
\hline J1023 State & H statistic & $\begin{array}{c}2 \sigma \text { pulsed VHE } \\
\text { flux UL }\left(\mathrm{m}^{-2} \mathrm{~s}^{-1}\right)\end{array}$ & $\begin{array}{c}3 \sigma \text { pulsed VHE } \\
\text { flux UL }\left(\mathrm{m}^{-2} \mathrm{~s}^{-1}\right)\end{array}$ \\
\hline $\begin{array}{c}\text { Radio MSP } \\
\text { Accretion/LMXB }\end{array}$ & 0.28 & $6.16 \times 10^{-9}$ & $9.73 \times 10^{-9}$ \\
\hline
\end{tabular}

Table 1: $H$ statistics and integral pulsed VHE flux upper limits $>166 \mathrm{GeV}$ for both the radio MSP and accrection/LMXB states of PSR J1023+0038.

The search for pulsed VHE emission was split into two parts as VERITAS data were taken both before and after the 2013 June disappearance of the radio pulsar (henceforth these two states of the system are referred to as the 'radio MSP' and 'accretion/LMXB' states). The VERITAS live time accumulated on the target is $18.1 \mathrm{hr}$ for the radio MSP state and $8.2 \mathrm{hr}$ for the accretion/LMXB state. The data span two configurations of the VERITAS array: before and after the upgrade of the camera photomulitplier tubes in the Summer of 2012 [34]. Events are phase-folded in Tempo 2 using a Jodrell Bank radio ephemeris (A. Archibald, private communication), though the location of phase zero is unknown, precluding the possibility of defining signal regions in the phaseogram. Therefore only the $H$-Test is used to test for the presence of a periodic signal in the data. The $H$-Test does not reveal any evidence for periodicity in the VHE phaseograms, which are shown in Figure 3. The $H$ statistics are used to compute 2 and $3 \sigma$ upper limits via the method of [35] above an energy threshold of $166 \mathrm{GeV}$ assuming

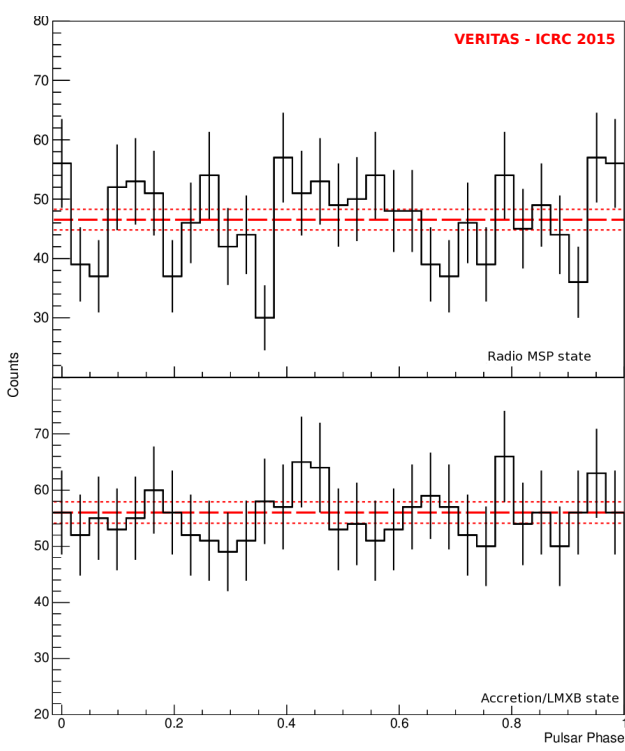

Figure 3: Phase-folded light curves of events recorded by VERITAS from the direction of PSR $\mathrm{J} 1023+0038$. One rotation of the pulsar is shown. The top panel shows the data collected during the radio MSP state of the system, while the bottom panel shows the data collected during the accretion/LMXB state. The solid and dashed lines represent the average and error on the average number of counts, respectively. a duty cycle of $10 \%$ and Gaussian pulse shapes. The computed $H$ statistics and pulsed flux ULs are shown in Table 1. A search for steady emission $>300 \mathrm{GeV}$ in the data is also performed, giving significances of $-0.8 \sigma$ and $0.2 \sigma$ for the radio MSP and accretion/LMXB states, respectively. Upper limits on a steady emission component are computed at the $95 \% \mathrm{CL}$ for both states assuming a photon index of $\Gamma=2.5$ using the Rolke method [36]. The computed steady flux 95\% CL ULs are $8.1 \times 10^{-9} \mathrm{~m}^{-2} \mathrm{~s}^{-1}$ and $9.6 \times 10^{-9} \mathrm{~m}^{-2} \mathrm{~s}^{-1}$ for the radio MSP and accretion/LMXB states, respectively. These results are part of a VERITAS collaboration publication that is currently in preparation. 


\section{Future Plans}

Pulsar science with VERITAS is an ongoing effort and will continue in the coming years. There are currently 19 pulsar locations for which VERITAS data exist, including coverage of the top ten Fermi-LAT detected pulsars in the Northern Hemisphere when ranked in spin-down power divided by distance squared (for details, see Archer, A., these proceedings) [37]. The analyses of these archival data sets is currently underway and will represent the first time that any of these pulsars have been probed for possible pulsed VHE emission.

Due to the good coverage of the young pulsars in archival VERITAS data, VERITAS is beginning to target a different promising population of pulsars to probe for VHE emission: millisecond pulsars. Because of the rapid rotation of MSPs (spin periods reaching down to $\sim 1 \mathrm{~ms}$ ), these pulsars have much smaller light cylinder radii, implying more compact magnetospheres. The compactness of their magnetospheres suggests significantly different physical parameters of the emission mechanism compared to the young pulsar population. Furthermore, though less luminous, their HE gamma-ray producion efficiencies have been found to be comparable or greater than those of the young pulsars [2]. For these reasons, targeting MSPs in addition to young pulsars should provide complementary benefits for constraining models attempting to explain their gamma-ray emission.

\section{Acknowledgements}

This research is supported by grants from the U.S. Department of Energy Office of Science, the U.S. National Science Foundation and the Smithsonian Institution, by NSERC in Canada, by Science Foundation Ireland (SFI 10/RFP/AST2748). We acknowledge the excellent work of the technical support staff at the Fred Lawrence Whipple Observatory and at the collaborating institutions in the construction and operation of the instrument. The VERITAS Collaboration is grateful to Trevor Weekes for his seminal contributions and leadership in the field of VHE gamma-ray astrophysics, which made this study possible.

\section{References}

[1] Cheng, K. S., Ho, C., \& Ruderman, M., Energetic Radiation from Rapidly Spinning Pulsars. II. VELA and Crab, ApJ, 300, 522 (1986)

[2] Abdo, A. A., Ajello, M., Allafort, A., et al., The Second Fermi Large Area Telescope Catalog of Gamma-Ray Pulsars, ApJS, 208, 17 (2013)

[3] Abdo, A. A., Ackermann, M., Atwood, W. B., et al., Fermi Large Area Telescope Observations of the Vela Pulsar, ApJ, 696, 1084 (2009)

[4] Gajdus, M., Pulsations from the Vela pulsar down to $30 \mathrm{GeV}$ with H.E.S.S. II, in proceedings of 34th International Cosmic Ray Conference (2015)

[5] Leung, G. C. K., Takata, J., Ng, C. W., et al., Fermi-LAT Detection of Pulsed Gamma-Rays above 50 GeV from the Vela Pulsar, ApJL, 797, L13 (2014)

[6] Aleksić, J., Alvarez, E. A., Antonelli, L. A., et al., Observations of the Crab Pulsar between 25 and 100 GeV with the MAGIC I Telescope, ApJ, 742, 43 (2011)

[7] Aliu, E., Arlen, T., et al., Detection of Pulsed Gamma Rays Above 100 GeV from the Crab Pulsar, Science, 334, 69 (2011)

[8] Lyutikov, M., Otte, N., \& McCann, A., The Very High Energy Emission from Pulsars: A Case for Inverse Compton Scattering, ApJ, 754, 33 (2012) 
[9] Du, Y. J., Qiao, G. J., \& Wang, W., Radio-to-TeV Phase-resolved Emission from the Crab Pulsar: The Annular Gap Model, ApJ, 748, 84 (2012)

[10] Lyutikov, M., The $\gamma$-Ray Spectrum of Geminga and the Inverse Compton Model of Pulsar High-energy Emission, ApJ, 757, 88 (2012)

[11] Aharonian, F. A., Bogovalov, S. V., \& Khangulyan, D., Abrupt acceleration of a 'cold' ultrarelativistic wind from the Crab pulsar, Nature, 482, 507 (2012)

[12] Pétri, J., High-energy emission from the pulsar striped wind: a synchrotron model for gamma-ray pulsars, MNRAS, 424, 2023 (2012)

[13] Mochol, I., \& Pétri, J., Very high energy emission as a probe of relativistic magnetic reconnection in pulsar winds, MNRAS, 449, L51 (2015)

[14] Caraveo, P. A., Bignami, G. F., Mignani, R., \& Taff, L. G., Parallax Observations with the Hubble Space Telescope Yield the Distance to Geminga, ApJL, 461, L91 (1996)

[15] Faherty, J., Walter, F. M., \& Anderson, J., The trigonometric parallax of the neutron star Geminga, Ap\&SS, 308, 225 (2007)

[16] Bignami, G. F., \& Caraveo, P. A., Geminga: Its Phenomenology, Its Fraternity, and Its Physics, ARA\&A, 34, 331 (1996)

[17] Bertsch, D. L., Brazier, K. T. S., Fichtel, C. E., et al., Geminga: new period, old $\gamma$-rays, Nature, 357, 306 (1992)

[18] Fichtel, C. E., Hartman, R. C., Kniffen, D. A., et al., High-energy gamma-ray results from the second small astronomy satellite, ApJ, 198, 163 (1975)

[19] Bennett, K., Lichti, G. G., Bignami, G. F., et al., COS-B observations of localised high-energy gamma-ray emission from the anticentre region of the galactic disc, $A \& A, \mathbf{5 6}, 469$ (1977)

[20] Kargaltsev, O. Y., Pavlov, G. G., Zavlin, V. E., \& Romani, R. W., Ultraviolet, X-Ray, and Optical Radiation from the Geminga Pulsar, ApJ, 625, 307 (2005)

[21] Halpern, J. P., \& Holt, S. S., Discovery of soft X-ray pulsations from the gamma-ray source Geminga, Nature, 357, 222 (1992)

[22] Deller, A. T., Archibald, A. M., Brisken, W. F., et al., A Parallax Distance and Mass Estimate for the Transitional Millisecond Pulsar System J1023+0038, ApJL, 756, L25 (2012)

[23] Archibald, A. M., Stairs, I. H., Ransom, S. M., et al., A Radio Pulsar/X-ray Binary Link, Science, 324, 1411 (2009)

[24] Thorstensen, J. R., \& Armstrong, E., Is FIRST J102347.6+003841 Really a Cataclysmic Binary?, AJ, 130, 759 (2005)

[25] Woudt, P. A., Warner, B., \& Pretorius, M. L., High-speed photometry of faint cataclysmic variables - IV. V356 Aql, Aqrl,

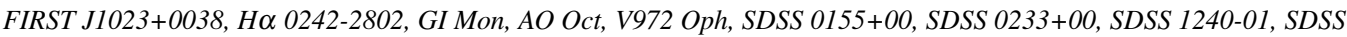
1556-00, SDSS 2050-05, FH Ser, MNRAS, 351, 1015 (2004)

[26] Stappers, B. W., Archibald, A., Bassa, C., et al., State-change in the "transition" binary millisecond pulsar J1023+0038, The Astronomer's Telegram, 5513, 1 (2013)

[27] Halpern, J. P., Gaidos, E., Sheffield, A., Price-Whelan, A. M., \& Bogdanov, S., Optical Observations of the Binary MSP J1023+0038 in a New Accreting State, The Astronomer's Telegram, 5514, 1 (2013)

[28] Alpar, M. A., Cheng, A. F., Ruderman, M. A., \& Shaham, J., A new class of radio pulsars, Nature, 300, 728 (1982)

[29] Holder, J., Acciari, V. A., Aliu, E., et al., Status of the VERITAS Observatory, American Institute of Physics Conference Series, 1085, 657 (2008)

[30] Hobbs, G. B., Edwards, R. T., \& Manchester, R. N., TEMPO2, a new pulsar-timing package - I. An overview, MNRAS, 369, 655 (2006)

[31] Aliu, E., Archambault, S., Archer, A., et al., A Search for Pulsations from Geminga above $100 \mathrm{GeV}$ with VERITAS, ApJ, 800, $61(2015)$

[32] de Jager, O. C., Raubenheimer, B. C., \& Swanepoel, J. W. H., A poweful test for weak periodic signals with unknown light curve shape in sparse data, $A \& A, \mathbf{2 2 1}, 180$ (1989)

[33] Helene, O., Upper limit of peak area, Nuclear Instruments and Methods in Physics Research, 212, 319 (1983)

[34] Kieda, D., et al., Status of the VERITAS Upgrade, in proceedings of 32nd International Cosmic Ray Conference, 9, 14 (2011)

[35] de Jager, O. C., On periodicity tests and flux limit calculations for gamma-ray pulsars, ApJ, 436, 239 (1994)

[36] Rolke, W. A., \& López, A. M., Confidence intervals and upper bounds for small signals in the presence of background noise, Nuclear Instruments and Methods in Physics Research A, 458, 745 (2005)

[37] Archer, A., Search for pulsed emission in archival VERITAS data, in proceedings of 34th International Cosmic Ray Conference (2015) 